\title{
Diagnostic Value of Qtc Dispersion and Qt Dispersion Ratio Changes on Stress Test in Detecting Significance of Coroner Lesion in Stable Angina Pectoris Patients
}

\author{
Putri Yeantesa 1, Hauda El Rasyid2, Masrul Syafri2, Ricvan Dana Nindrea 3
}

\begin{abstract}
Background: Treadmill exercise test remains an important method and often used in the initial evaluation of patients with chest pain and can be a filter for more expensive invasive diagnostic. Increased QT dispersion (QTD) occurs because of the heterogeneity of ventricular repolarization because transient ischaemia during a treadmill stress test can be a marker of coronary artery disease (CAD) and can improve the accuracy of exercise tests to diagnose CAD, but this parameter is still controversial.

Methods: This is an analytic observational approach with a cross sectional study. Data was taken retrospectively at the Heart Center Installation at RSUP Dr. M. Djamil Padang, from March to April 2019, stable angina pectoris patient with a positive treadmill stress test who underwent coronary angiography as the subject. Bivariate analysis was performed on changes in $\mathrm{QTcD}(\triangle \mathrm{QTcD})$ and $\mathrm{QTdR}(\triangle \mathrm{QTdR})$ variables on the significance of coronary lesions by the chi-square method, after which a diagnostic test was based on receiver operating curve (ROC) analysis.

Results: There were 122 subjects and found that older age, male and smoking were more common in groups with significant coronary lesions. Cut off point for $\triangle Q T C D$ is $\geq 13$ ms with a sensitivity of $87,1 \%$ and specificity $85,2 \%$ and $A U C$ 95 , $1 \%$, while $\triangle Q T d R \geq 5.5 \%$ with sensitivity $85,7 \%$ and specificity $81,9 \%$ and AUC $90,5 \%$ are related to significancy of coronary lesion. Obtained subjects with significant lesions generally had a value of $\Delta Q T c D \geq 13 \mathrm{~ms}(\mathrm{p}<0.00 \mathrm{I})$ and $\triangle \mathrm{QTdR} \geq 5.5 \%(\mathrm{p}<0.00 \mathrm{I})$.

Conclusion: The use of $\triangle \mathrm{QTdR}$ dan $\triangle \mathrm{QTdR}$ parameters as ECG variables, which are easily obtained in evaluating stress tests, can improve the diagnostic accuracy of exercise tests. In addition, evaluation of $\Delta \mathrm{QTdR}$ dan $\Delta \mathrm{QTdR}$ can provide information about the incidence of CAD.
\end{abstract}

(Indonesian J Cardiol. 2019;40:262-268)

Keywords : $\triangle \mathrm{QTCD}, \Delta \mathrm{QTdR}$, Treadmill Exercise Test, Coronary Lession Significancy

I Faculty of Medicine, University of Andalas, West Sumatera, Indonesia;

2 Department of Cardiology and Vascular Medicine, M Djamil Hospital, West Sumatera, Indonesia;

3 Department of Public Health and Community Medicine Faculty of Medicine, University of Andalas, West Sumatera, Indonesia.

\section{Correspondence:}

dr Putri Yeantesa

Faculty of medicine, Universitas Andalas

E-mail:yeantesa@yahoo.com

\section{Introduction}

7 he treadmill test (TMT) is the most widely used test for the initial evaluation of patients with chest pain. In addition to ease of use, safety, relatively low cost and relatively fast examination, TMT is a filter towards more expensive and invasive examination. ${ }^{1}$ However, the sensitivity of the stress test with ECG using the ST segment depression criteria was not very good, only $45-50 \% .^{2}$ 
An informative electrocardiographic indicator for diagnosing coronary heart disease during stress test is well known. ${ }^{3}$ The ventricular repolarization process caused by ischemia, which is represented by ST segment changes or T-wave inversion has been widely used in the study of patients with coronary heart disease. Some studies show QT dispersion (QTd) and QT dispersion ratio $(\mathrm{QTdR})$ are significant predictors of cardiovascular mortality although there are still many controversial results. ${ }^{4}$

Increased QTd values can be found in patients with stable angina pectoris. There are several studies which state that there is a relationship between elongation of QTd and QTdR values in patients with stable angina pectoris both at the time of recording ECG 12 leads at rest and at the peak of exercise test with significance or severity of lesions in coronary arteries. Addition of the parameters $\triangle \mathrm{QTCD}$ and $\triangle \mathrm{QTdR}$ at the time TMT can provide better sensitivity to detect ischemic responses in patients with stable angina pectoris than only using conventional criteria that have been previously set. $5-8$

\section{Methods}

This is an observational analytic approach with a cross sectional study design. All data were taken retrospectively from the medical records of patients with stable angina pectoris (SAP) at RSUP Dr. M. Djamil Padang from March to April 2019. The inclusion criteria were SAP patients who had a TMT with a positive ischemic response and carrying out coronary angiography. Exclusion criteria were age > 75 years, diabetes mellitus, heart failure with decreased of left ventricular ejection fraction, rpacing rhythm, history of myocardial infarction, valvular heart disease, arrhythmia / use of class I and III anti-arrhythmic drugs, Long QT syndrome, electrolyte disorders, history PCI / CABG, ECG is difficult to assess. Demographic data were collected, followed by EKG at rest and peak TMT and coronary angiography results. Demographic data including age, sex, and treatment history. QTc dispersion $(\mathrm{QTcD})$ is the longest $\mathrm{QTc}$ value minus the shortest QTc of 12 EKG leads, while the QT dispersion ratio is $\mathrm{QTcD}$ divided by cycle length, then the $\mathrm{QTcD}$ changes $(\Delta \mathrm{QT} c \mathrm{D})$ and $\mathrm{QTdR}$ changes $(\Delta \mathrm{Q} T \mathrm{Td})$ were measured in resting and peak TMT. Assessment of $\triangle \mathrm{QTcD}$ and $\Delta \mathrm{QTdR}$ using ECG recorded data printed from treadmill monitor was carried out by observers who were experienced and blind to clinical data and the patient's coronary angiography results. After that, an assessment of coronary angiography was also performed by observing the presence of significant coronary lesions. Statistical analysis was performed to see the relationship between independent and dependent variables using the chi-square test with a confidence degree of $95 \%$ and $\alpha$ $=0.05$. If the value of $p<0.05$ means that there is a significant relationship between the statistics studied. The diagnostic test between $\triangle \mathrm{QTcD}$ and $\Delta \mathrm{QTdR}$ in SAP patients who have positive TMT results with significance of coronary lesions is based on receiver operating curve (ROC) analysis that has an area under curve (AUC) value greater than or equal to the expected AUC value. The sensitivity and specificity based on the cut-off point will be tested according to Receiver Operating Characteristic (ROC) analysis. Data were analyzed using the Statistical Package for Social Sciences (SPSS) program.

\section{Results}

Total 154 subjects who met the inclusion criteria, 32 subjects were excluded for various reasons as listed in Figure 1. There were 70 subjects with significant coronary lesions, total of one, two and three coronary artery disease (CAD) was 18 (26\%), 43 (61\%) and 9 (13\%) subjects consecutively. The group with nonsignificant lesions found $40(77 \%)$ subjects with normal coronary arteries and $12(23 \%)$ people with non-significant lesions. The group of significant lesions also had more risk factors for smoking, dyslipidemia, and hypertension than the group with non-significant lesions. Antiplatelet was used in almost all groups with significant lesions, and the use of Angiotensin Converting Enzyme inhibitors (ACE-I), beta blockers, statins, and glyceril trinitrate mostly used in group with significant lesions and statistically different between two groups. This value can be seen in table 1 .

There were no significant differences in the QTc, QTcDr, and QTdRr values in the two groups of patients, but QTcDs and QTdRs were significantly longer in patients with significant lesions than the non-significant lesion group. The mean of $\triangle \mathrm{QTCD}$ in the group of 
significant coronary lesions was $27.87 \pm 13.39$ ms higher than non-significant $7.44 \pm 13.63 \mathrm{~ms}$. While the mean of $\triangle \mathrm{QTdR}$ in the group of significant coronary lesions was $9.83 \pm 4.45 \%$ compared to non-significant $4.56 \pm$ 3.36 , the results obtained were statistically difference.

The cut off point of $\triangle \mathrm{QTCD}$ is $13 \mathrm{~ms}$ and $\triangle \mathrm{QTdR}$ is $5.5 \%$, so the value of $\Delta \mathrm{QTcD} \geq 13 \mathrm{~ms}$ and $\Delta \mathrm{QTdR}$ $\geq 5.5 \%$ were prominently higher value. The cut off point of $\triangle \mathrm{QTcD}$ and $\triangle \mathrm{QTdR}$ as well as sensitivity and specificity can be seen in table 2. Tables 3 and 4 showed occurrence of coronary lesions significantly more in subjects with higher $\Delta \mathrm{QTcD}(87.1 \%)$ than normal $(3.8 \%)$ and after the chi-square statistical test, $\mathrm{p}$ value $=<0.001$ was obtained. The incidence of significant coronary lesions was also greater in subjects with prolonged $\triangle \mathrm{QTdR}(85.7 \%)$ compared to normal (3.8\%) and after chi-square statistical tests were obtained $\mathrm{p}=<0.001$. So there were relationship between $\Delta \mathrm{QTcD}$ and $\triangle \mathrm{QTdR}$ and coronary lesions in patients with stable angina pectoris ( $\mathrm{p}$ value $<0.05$ ).

\section{Discussion}

Men were mostly found in group with significant lesion between SAP patients with positive TMT results who underwent coronary angiography. Whereas in groups with non-significant lesions dominated by women. This is in line with the population study conducted by Cheng, that men with typical angina have a greater risk of suffering from coronary heart disease with lesions that are heavier than women $(19 \%$ vs $11 \%$ with $p$ $<0.001) .{ }^{9}$ In a population study from Finland involving 11,834 male and female subjects aged 35-52 years who were followed up for 12 years, it was found that the incidence of myocardial infarction was 4.6 times greater in men than in women. ${ }^{10}$ More men were affected by the disease are associated with unhealthy behaviors such as smoking, alcohol consumption, lack of vegetable and fruit consumption and exposure to stressors that are socially more acceptable for men than women. ${ }^{11}$ In addition, the protective cardio effects of estrogen may also play an important role in their low female subjects suffering from coronary artery disease. Favorable changes of plasma lipid profiles, protective effects directly into the vascular wall, the role of estrogen as an antioxidant, ability to protect from DNA damage, inhibition of cell proliferation and prevent vascular responses to vasoactive agents are the cardio protective effects of estrogen. ${ }^{12}$ Older age is also more common in groups with significant lesions. Old age is a cardiovascular risk factor that cannot be modified. This is related to the aging process which causes a decrease in the function of organs. ${ }^{13}$

This study showed more women in the group with non-significant lesions, this means a high number of false positives from the results of treadmill in women. In a meta-analysis, it was found that ST segment depression in women had significantly lower specificity compared to men. False positive rates in women based on disease prevalence range from $25 \%$ in patients with typical angina to $50 \%$ in patients with atypical chest pain. Several mechanisms have been expressed to explain the high false positive rate of ST-segment depression in women, including the fact that estrogen has a chemical structure similar to digitalis during exercise, this is responsible for the high prevalence of false-positive test results in women and causes differences in accuracy of ST segment depression in women compared to men. ${ }^{7}$

Antiplatelet was used in almost all subjects with significant lesions and only $44.2 \%$ in the group with insignificant lesions. Beta blockers, and statins are also used in almost all subjects with significant lesions, because the use of beta blockers in cases of CAD is needed for anti-ischemic and statin for stabilisation of plaque. The use of Angiotensin Converting EnzymeInhibitors (ACE-I), and glyceryl trinitrate are also numerous in groups with significant lesions. Significant differences between the two groups on drug use are possible because in the group with significant lesions there were 34 patients who had entered the emergency department (ED) with a diagnosis of stable angina pectoris with low risk stratification and normal ECG patterns then discharge and advised for stress test from the out patient clinic, whereas in the group with nonsignificant lesions there were only 7 patients who had entered the ED with the same diagnosis. Individuals with angina pectoris do not experience myocardial necrosis, have a substantially lower risk of death and seem to had less benefit from invasive strategies. ${ }^{14}$ Therefore this is the role of stress tests in patients with a history of angina pectoris to determine whether patients should be referred for invasive diagnostics or not. 
The values of QTcDs, QTdRs, $\triangle \mathrm{QTcD}$ and $\triangle \mathrm{QTdR}$ in patients with significant coronary lesions group were found to be higher compared to nonsignificant lesion group. This was in accordance with the study by Ozdemir and Piranfar who obtained this QT parameter to be extended in patients with significant coronary lesions. This is due to the heterogeneity of ventricular repolarization that occurs in the heart muscle that experiences ischaemia during exercise test. Possible mechanisms for prolonged QT dispersion in patients with coronary artery disease appear to be caused by electrolytes and metabolic imbalances such as increased extracellular potassium and decreased $\mathrm{pH}$ due to accumulation of lactic acid. Changes in ionic balance throughout the sarcolemma lead to an extension of the duration of repolarization and delay in conduction which causes a dispersion of the duration of the action potential in ischemic ventricular myocardial cells leading to increased QT dispersion. So that why QT dispersion during stress will be longer than at rest in patients with significant coronary lesions. ${ }^{7,15}$ In exercise QTd prolongation is positively related to the number of schematic segments and the number of ischemic scores measured by isotope scan in all patients with significant coronary lesions, whereas in normal patients QTd rest and stress have values that are not significantly different. The QTd value of the exercise is related to the severity and amount of myocardial ischaemia and is clinically useful for the identification of myocardial ischemic injury. 8,12

In this study the cut off point for $\triangle \mathrm{QTcD}$ in predicting coronary lesions was $13 \mathrm{~ms}$, so that $\Delta \mathrm{QTcD}$ $<13 \mathrm{~ms}$ became normal value and $\Delta \mathrm{QTcD} \geq 13 \mathrm{~ms}$ became prolonged value. These results have a sensitivity of $87.1 \%$ and specificity of $85.2 \%$, and have very good accuracy $(95.1 \%)$ based on the AUC value. These results similar with Ozdemir's which states that there was a relationship between $\Delta \mathrm{QTCD}$ and the presence of coronary lesions and $\triangle \mathrm{QTCD}$ that are sensitive and specific for diagnosis of CAD with a value of $84 \%$ and $88 \%$. In study by Ozdemir, the value of the crosssectional point is higher, which is $\Delta \mathrm{QTCD} \geq 15 \mathrm{~ms}$ as the prolonged value. ${ }^{7}$

Another study that is also in line with this research is the study by Pshenichnikov, who stated that prolonged $\Delta$ QTcD ( $\geq 20$ mdet) was independently associated with poor prognostic in patients with stable angina pectoris.
In this study assessing the prognostic of stable angina pectoris patients who underwent a stress test in $36 \pm 12$ months, it was found that the group that experienced advanced cardiovascular events had a value of $\Delta \mathrm{QTcD}$ that was prolonged compared to the stable group or experienced clinical improvement. In this study, it was found that prolonged $\triangle \mathrm{QTcD}$ ( $\geq 20 \mathrm{mdet}$ ) in stable angina pectoris patients was associated with advanced cardiovascular events and had a sensitivity of $77.3 \% \%$ and specificity $78.8 \%$ and if the parameter $\triangle \mathrm{QTcD}$ was combined with ST segment depression criteria during the stress test the sensitivity and specificity up to $93.8 \%$ and $66.7 \% .{ }^{16}$

The cut off value in this study is smaller compared to previous studies. The profile of coronary lesions in patients with significant coronary lesions in this study was there were more patients with two-vessel CAD, 43 (61\%) of 70 patients but the lesion located in mid or distal vessels. In the Psenichnikov study, the increased in $\mathrm{QTCD}$ was more related to the degree, location and extent of coronary artery lesions. Subocclusion in LAD before the first diagonal artery (high risk injury) often reflects a greater increase in $\mathrm{QTcD}$ value compared with multivessel disease with distal coronary artery lesions (moderate risk injury). The extent of the heart muscle that experiences ischaemia in lesions in LAD is the cause of greater value of $\triangle \mathrm{QTcD} .{ }^{17}$ Therefore why the value of $\Delta \mathrm{QTCD}$ in this study was lower than previous studies.

The sensitivity and specificity in this study were quite good, having values similar to those obtained by Ozdemir's. The subjects with CAD two vessels was the biggest number with 43 subjects (61\%), while CAD one and three vessel were only $18(26 \%)$ and 9 subjects (13\%) respectively. Of the nine subjects with CAD 3 vessels, the prolonged value of $\triangle \mathrm{QTcD}$ was obtained by only two subjects, while the others had normal $\triangle \mathrm{QTCD}$ values. False negative results make it possible to occur in subjects with three vessels CAD where the ischemic events that occur in the entire heart muscle due to significant stenosis from all three epicardial vessels can cause no significant QTcD changes between rest and exercise due to QTc values in all EKG leads will prolonged so that the dispersions will be small and changes between rest and exercise are also small.

In this study, the cut off point for $\triangle \mathrm{QTdR}$ in predicting coronary lesions was $5.5 \%$, so that $\Delta \mathrm{QTdR}$ $<5.5 \%$ became normal value and $\Delta \mathrm{QTdR} \geq 5.5 \%$ 
became prolonged $\triangle \mathrm{QTdR}$. These results have a sensitivity of $85.7 \%$ and specificity of $81.9 \%$, and have very good accuracy $(90.8 \%)$ based on the AUC value (Figure 2). This result is in line with Ozdemir's research which states that there is a relationship between $\Delta \mathrm{QTdR}$ and the presence of coronary lesions and $\triangle \mathrm{QTdR}$ which are sensitive and specific for diagnosis of CHD with a sensitivity value of $84 \%$ and specificity of $96 \%$. In this study by Ozdemir, the cross-sectional point values were slightly lower, namely $\Delta \mathrm{QTdR} \geq 5 \%$, which was a longitudinal value, but this study was only carried out in female patients and there were no studies that assessed $\triangle \mathrm{QTdR}$ in male and female patients.7 If we analysed value of $\triangle \mathrm{QTdR}$ in subjects with three blood vessels $\mathrm{CAD}$ only prolonged in two subjects, seven other have normal $\triangle \mathrm{QTdR}$ values. In contrast to subjects who have $\mathrm{CAD}$ one or two vessels, almost all of them have an $\triangle \mathrm{QTdR}$ value that is prolonged. Possible underlying causes are the same as in the $\Delta \mathrm{QTCD}$ parameter above, namely the existence of balance ischemia which causes false negatives.

Previous study by Sahin found that QTdR was superior to $\mathrm{QTcD}$ for diagnosing the incidence of ventricular fibrillation, but this parameter is still rarely used in studies that assess ECG parameters to detect coronary artery disease. This study by Sahin assessed the relationship of QTdR parameters with stable angina pectoris. It was found that patients who had significant coronary lesions had significantly longer QTdR values compared to patients with normal coronary but this parameter was only assessed at rest, there was no comparison with exercise ECG. There is only one study that assesses the parameters of $\triangle \mathrm{QTdR}$ in stable angina pectoris patients who carry out the stress test, namely the study conducted by Ozdemir and the results obtained did not differ much from this study, where Ozdemir scored $\Delta \mathrm{QTdR} \geq 5 \%$ indicating coronary artery disease. ${ }^{6,7}$

In the group with significant lesions in this study have prolonged $\triangle \mathrm{QTCD}$ value $(87.1 \%)$, and the difference was statistically significant between the nonsignificant group $(\mathrm{p}<0.001)$. In line with these results, the prolonged $\triangle \mathrm{QTdR}(85.7 \%)$ also had a statistically significant difference $(\mathrm{p}<0.001)$ between patients with significant and non-significant coronary lesions. Based on these results it was found that there was a relationship between $\Delta \mathrm{QTCD}$ and $\triangle \mathrm{QTdR}$ with coronary lesions in stable angina pectoris patients. The possible mechanism for increasing QT dispersion in patients with coronary artery disease involves the mechanism of prolongation of the QTc interval in conditions of ischemia involving the presence of electrical heterogeneity, acidosis, lysophosphatidylcholine and electrolyte imbalance. Changes in ionic balance throughout myocardial cells cause an extension of the duration of the action potential in the muscle that experiences ischemia so that the duration of the action potential in ventricular myocardial cells is ischemic and causes an increase in dispersion QT. ${ }^{15,17}$

The Koide et al study concluded that QTd values at peak exercise were independent predictors for determining the presence or absence of significant coronary artery stenosis. The sensitivity and specificity of TMT in this study were $76 \%$ and $86 \%$. When added to QTd at the peak of exercise more than $60 \mathrm{~ms}$, the sensitivity of the stress test increased to $84 \%$ while the specificity increased to $90 \%$. Aytemir et al evaluated the benefits of a combination of QTd and ST segment depression during a TMT to predict the incidence of re-stenosis after percutaneous coronary intervention. Sensitivity and specificity of QTd $\geq 60$ ms for prediction of re-stenosis were $83 \%$ and $61 \%$. When ST segment depression is added, sensitivity and specificity increase to $91 \%$ and $78 \% .18,19$

Study by Bagus in 2015 showed a positive relationship between changes in QT dispersion and the severity of coronary lesions assessed using the Syntax score. The value of $\Delta \mathrm{QTd} 44.78 \mathrm{~ms}$ is the optimal discriminating point between high and low Syntax scores. ${ }^{5}$

Study by Sahin reported a correlation between ECG parameters and the presence and extent of CAD to demonstrate the usefulness of this parameter as a predictor of the severity of coronary lesions in patients with stable CAD. From this study, QTcd and QTdR were lowest in the normal coronary group and the longest in the group with three vascular coronary lesions. In this study, QTdR was the best ECG parameter for detecting significant and not significant coronary artery narrowing (AUC 0.858). ${ }^{6}$

In this current study most of the patients with CAD three vessels had a value of $\triangle \mathrm{QTcD}$ and $\triangle \mathrm{QTdR}$ within normal limits. It is feared that patients with CAD three vessels can cause a balance of ischemia, because if there are significant lesions in the three blood vessels, then the probability at the peak stress test QTc values will increase 
in all ECG leads where QTcD and QTdR values will not differ significantly compared to QTcD and QTdR values at rest so that it can cause false negatives.

\section{Conclusion}

Risk factors such as men, old age and smoking habits were more common in patients with significant lesions. $\Delta \mathrm{QTcD} \geq 13 \mathrm{~ms}$ and $\Delta \mathrm{QTdR} \geq 5,5 \%$ indicated an prolonged of the QT parameter and had a good sensitivity and specificity, also excellent predictive ability to detect significant coronary lesions. The prolonged of these two parameters has a significant relationship with the significance of coronary lesions in angina pectoris patients undergoing a treadmill stress test.

\section{Ethical Clearance}

This study was approved by the institution's ethic committee.

\section{Conflict Of Interest}

None

\section{Publication Agreement}

The authors of this article give permission to Indonesian Journal of Cardiology to publish this article if this article is accepted

\section{Funding}

Self funded

\section{List of Abbreviations}

AUC : Area under curve

CVD : Cardiovascular disease

CAD : Coronary artery disease

ECG : Electrocardiography

LPC : Lysophosphatidylcholine

Ms : Mili secon

QTc : Corrected QT interval

QTd : QT dispersi

QTdR : QT dispersi rasio
QTcDr : QT dispersi rest

QTcDs : QT dispersi stress

QTdRr : QT dispersi rasio rest

QTdRs : QT dispersi rasio stress

TMT : Treadmill test

SYNTAX : Synergy between Percutaneous Coronary Intervention with Taxus and Cardiac Surgery

\section{References}

1. Mayasari E, Arso I, Maharani E. Diagnostic Value of Duke Treadmill Score in Predicting Coronary Lesions Severity in Patients with Suspected Stable Coronary Artery Diseases. J Kardiol Indones. 2016;37:65-74.

2. Radi B, Arso I, Sarvasti D, Tadjoedin Y, Tjahjono C. Pedoman Uji Latih Jantung: Prosedur dan Interpretasi. Jakarta: PERKI; 2016.

3. Helmy H, Abdel-Galeel A, Kishk Y, Sleem K. Correlation of corrected QT dispersion with the severity of coronary artery disease detected by SYNTAX score in non-diabetic patients with STEMI. The Egyptian Heart Journal. 2017.

4. De Bruyne M, Hoes A, Kors J, Hofman A, Van Bemmel J and Grobbee D. QTc Dispersion Predicts Cardiac Mortality in the Elderly: The Rotterdam Study. Circulation. 1998;97:467-72.

5. Pramono B, Maharani E, Irawan B. Relationship between QT Dispersion Changes on Treadmill Test with Coronary Lesion Degree in Patients with Suspected Stable Coronary Artery Disease. Jurnal Kardiologi Indonesia. 2016;37:122-9.

6. Şahin B, Poyraz E, Demirelli S, Yıldırım E, Ipek E, Cengiz M, Bayantemur M. The Relationship between P \& QT Dispersions and Presence \& Severity of Stable Coronary Artery Disease. Korean Circulation Journal. 2016;46(4):522-9.

7. Özdemir K, Altukeser B, Mustafaaydin, Aliozeren, Gulizardani S, HAasangok. New Parameters in the Interpretation of Exercise Testing in Women: QTc Dispersion and QT Dispersion Ratio Difference. Clin Cardiol 2002;25:187-92.

8. Assadpour Piranfar M. The Relationship between QT Dispersion and Ischemic Injuries in Myocardial Isotope Scan. Acta Medica Iranica. 2014;52(5):34551. 
9. Cheng V, Berman D, Rozanski A, Dunning A, Achenbach A, Al-Mallah M, et al. Performance of the Traditional Age, Sex, and Angina TypicalityBased Approach for Estimating Pretest Probability of Angiographically Significant Coronary Artery Disease in Patients Undergoing Coronary Computed Tomographic Angiography. Circulation. 2011;124:2423-32.

10. Zhang X, Patel A, Horibe H, Wu Z, Barzi F, Rodgers A Cholesterol, coronary heart disease, and stroke in the Asia Pacific region. International Journal of Epidemiology. 2003;32:563-72.

11. Rahajeng E, Tuminah S. Prevalensi Hipertensi dan Determinannya di Indonesia. Maj Kedokt Indon. 2009;59:580-7.

12. Subbiah MT. Estrogen replacement therapy and cardioprotection: mechanisms and controversies. Brazilian Journal of Medical and Biological Research. 2002;35(3):271-6.

13. Çağlar F, Çağlar L, Akturk F, Demir B, Yüksel Y, Firatli I. The Association between QT DispersionQT Dispersion Ratio and the Severity-Extent of Coronary Artery Disease in Patients with Stable Coronary Artery Disease. İstanbul Med J 2014;15: 95-100.

14. Roffi M, Patrono C, Collet JP, Mueller C, Valgimigli M, Andreotti F, et al. 2015 ESC Guidelines for the management of acute coronary syndromes in patients presenting without persistent ST-segment elevation. European Heart Journal 2016;37:267315.

15. Mubarak S, Majeed S, Khan M. QT Dispersion In Patients With Coronary Artery Disease. Pak J Physiol. 2015;11(1):7-9.

16. Pshenichnikov I, Shipilova T, Laane P, Meigas K, Anier A, Kaika J. Prognostic value of QT interval dispersion during exercise in patients with stable angina. Seminars in Cardiovascular Medicine. 2008;14:1-5.

17. Higham P, Furniss S, Campbell R. QT dispersion and components of the QT interval in ischaemia and infarction. Br Heart J. 1995;73:32-6.

18. Aytemir K, Necla B, Aksoyek M, Ozkutlu H, Ali M, Ozmen F. QT Dispersion plus ST-Segment Depression: A New Predictor of Restenosis after Successful Percutaneous Translurninal Coronary Angioplasty. Clin Cardiol. 1999;22:409-12.
19. Koide Y, Ukurma M, Kazuhdiot, Osmo H, Hika K. Use of QT Dispersion Measured on Treadmill Exercise Electrocardiograms for Detecting Restenosis after Percutaneous Transluminal Coronary Angioplasty. Clin Cardiol. 2001;22:63948. 